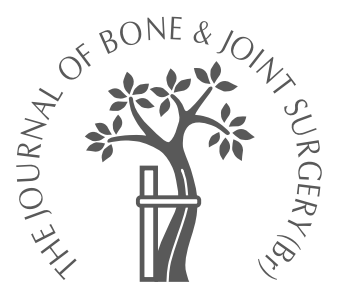

A. Arora, A. Agarwal

From University College of Medical Sciences and Guru Teg Bahadur Hospital, Delhi, India

A. Arora, MS, Reader A. Agarwal, MS, Senior Resident

Department of Orthopaedics, University College of Medical Sciences, Shahdara, Delhi 110095, India.

Correspondence should be sent to $\mathrm{Dr}$ A. Arora at F-4/9, First Floor, Mandir Marg Krishna Nagar, New Delhi 110051, India.

(C)2004 British Editorial Society of Bone and Joint Surgery doi:10.1302/0301-620X.86B2. $14227 \$ 2.00$

$J$ Bone Joint Surg $[\mathrm{Br}]$ 2004;86-B:282-4.

Received 31 January 2003;

Accepted after revision

16 July 2003

- CASE REPORTS

\title{
Dhaga syndrome: a previously undescribed entity
}

Three children between the ages of 18 months and four years presented with a discharging sinus or sinuses at the wrist due to a 'sacred' thread (the Moli Dhaga) which had been tied around it. This thread had been forgotten by the parents and had become embedded in the soft tissues of the wrist. Plain radiographs showed a circumferential constriction in the soft tissue shadow in all three. In two, there was a periosteal reaction in the distal radius or ulna with an indentation which we have called the constriction sign. Surgical removal of the buried thread was successful in all cases.

\section{Case reports}

Case 1. A girl aged 18 months presented with a discharging sinus on the volar aspect of the right wrist which had been present for three months (Fig. 1a). She had experienced repeated episodes of low-grade fever. There was no history of trauma or discharge of coloured granules. The right wrist was swollen, red, indurated and warm but no fluctuation could be elicited. There was an intermittent, foul-smelling, seropurulent discharge from the sinus. The hand was swollen and because of this both pulses were difficult to palpate. Active finger movements were present and the circulation and capillary filling of the fingers were normal. Although sensation was difficult to assess, the child responded to painful and tactile stimuli. One epitrochlear lymph node was enlarged but the axillary nodes were not. Routine blood tests were within normal limits except for the erythrocyte sedimentation rate which was raised to $40 \mathrm{~mm} / \mathrm{hour}$.

Plain radiographs showed a circumferential soft tissue constriction in the wrist region but the bones appeared normal (Fig. 2). Culture of the discharge grew Staphylococcus aureus. Fine needle aspiration cytology of the enlarged epitrochlear lymph node showed a non-specific inflammatory reaction. A clinical diagnosis of tuberculous infection of the wrist with secondary Staphylococcal infection was made. Standard multidrug anti-tubercular chemotherapy was prescribed and a below-elbow volar slab applied. ${ }^{1}$ The inflammation around the wrist decreased, but the discharging sinus persisted. Exploration was then undertaken through a horizontal incision on the volar aspect of the wrist. Careful dissection at the site of sinus revealed a knot of thread. On gently pulling this knot, there were movements of the soft tissue over the entire circumference of the wrist, indicating complete encirclement by the thread. The thread was dissected out of the scar tissue, superficial to the volar tendons, the knot was cut and the whole thread carefully pulled out. The skin lesions healed within three weeks after a course of antibiotics.

On careful questioning, the parents remembered the thread, which had been tied on the child's wrist, while performing a religious ceremony nearly six months previously. The red and yellow thread popularly known as Moli in the local language is tied around the wrist on important religious occasions. Case 2. A girl aged four years presented with multiple discharging sinuses at the right wrist for two months with a linear skin marking around the entire circumference of the wrist (Fig. 1b). There was no history of fever, apart from some swelling on the dorsum of the hand, and the neurovascular status of the hand was normal. Cultures were sterile. Radiographs revealed a longitudinal periosteal reaction on the ulnar side of the distal ulna with a characteristic indentation which we have called 'the constriction sign' (Fig. 3).

After careful questioning, no history of a thread being tied around the wrist in the past was forthcoming. We suspected an embedded thread which had been forgotten as in case 1, and explored the wrist through an S-shaped dorsal incision. The thread was localised on the ulnar border of the distal ulna, cut and slowly pulled out. The lesions healed within three weeks.

Case 3. A boy aged four years presented with a discharging sinus over the lateral aspect of the right wrist which had been present for two and a half years. The sinus was covered with a dry crust. The parents were unable to recall a thread being tied around the wrist. He had been previously treated with eight months of supervised anti-tubercular chemotherapy followed by unsuccessful exploration of the sinus by a local practitioner six months before presenting to us. There was a linear scar around the circumference of the wrist with a longitudinal scar from the previous operation on the dorsum (Fig. 1c). Examination of the hand and arm was otherwise normal. There was no lymph node enlargement in 


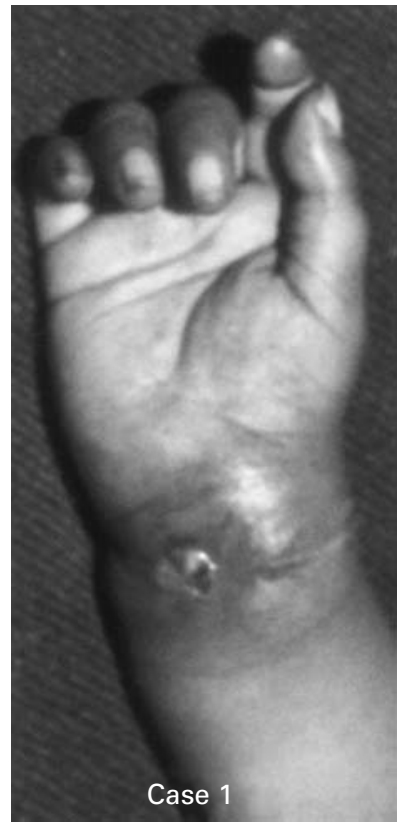

Fig. 1a

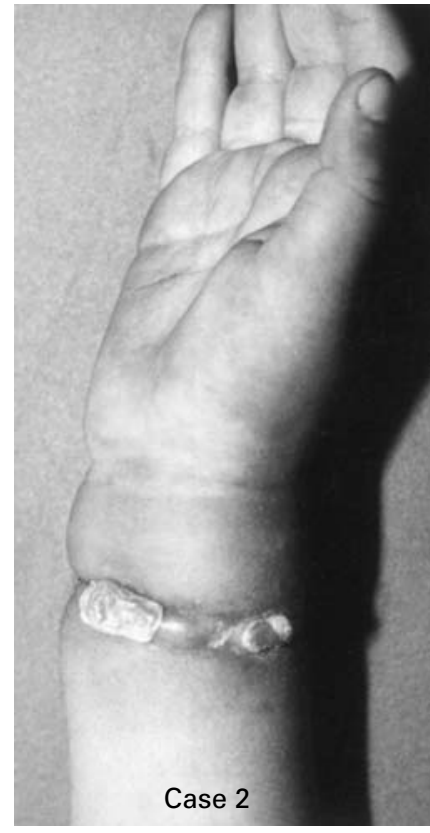

Fig. 1b

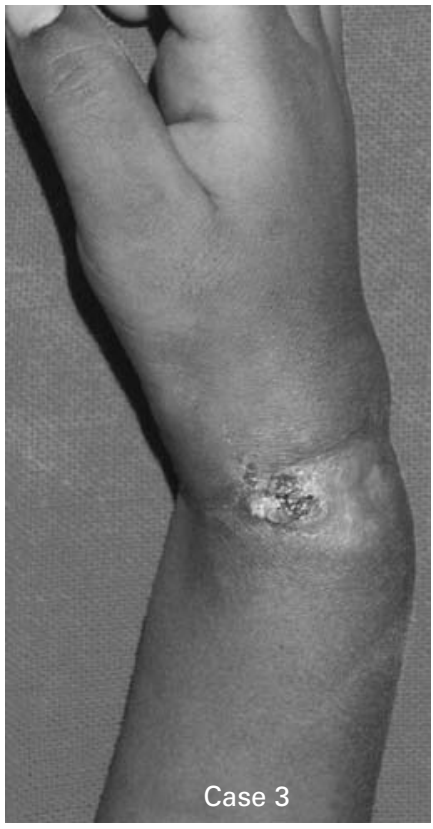

Fig. 1c

Photographs taken at the clinical presentation showing the discharging sinus in the three children.

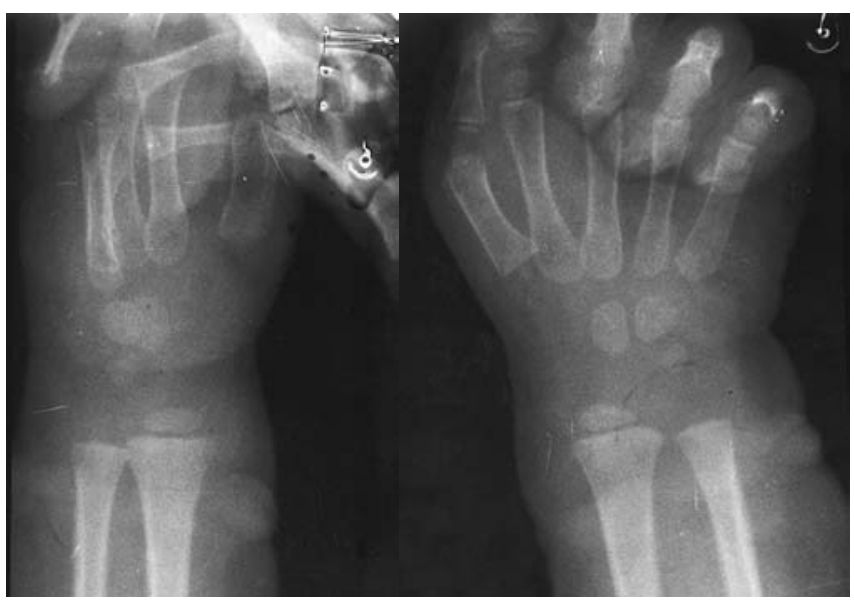

Fig. 2

Radiographic findings in case 1 showing a circumferential soft tissue shadow, although the bones appear normal.

the elbow or axillary region. On removal of the crust, a seropurulent discharge was present, which was sterile on culture. Anteroposterior plain radiographs of both wrists showed an indentation in the radial side of the right distal radial epiphysis, the 'constriction sign', and a circumferential constriction of the soft tissue of the right wrist (Fig. 4).

The wrist was explored through a lazy S-shaped incision. The thread had to be removed by dissection medially and laterally as it had been sectioned by the previous dorsal incision. It lay deep to
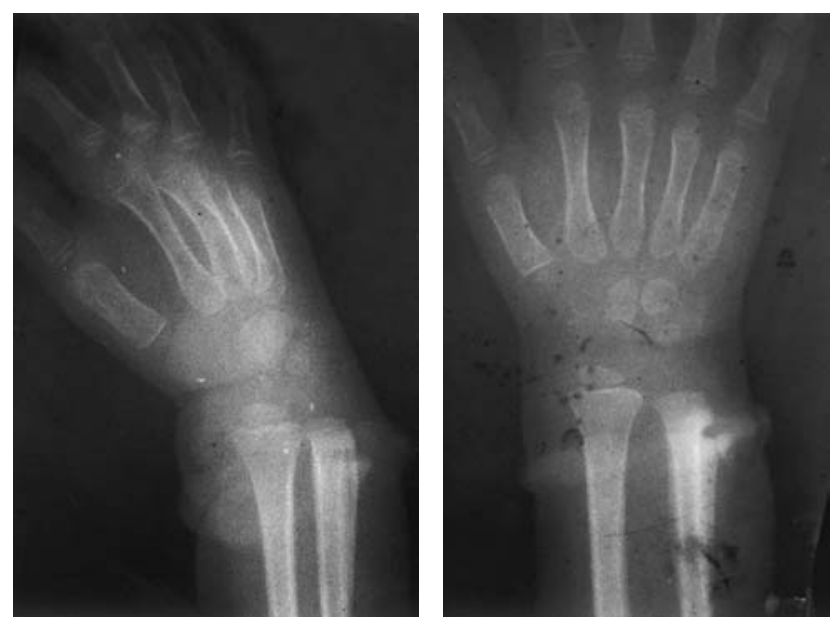

Fig. 3

Radiographic findings in case 2 showing the 'constriction sign', which appears as an indentation or constriction in the middle of the longitudinal periosteal reaction on the distal ulna.

the palmaris longus tendon and was partially buried in the tendon of flexor carpi ulnaris (Fig. 5). The lesion healed rapidly after surgery and treatment with antibiotics.

\section{Discussion}

In the Indian sub-continent, it is customary to tie a sacred thread (the Moli Dhaga in the Hindi language) on the wrist before any religious ceremony is begun. Self-removal of this sacred thread is considered an ominous sign, and so the thread may remain in situ 

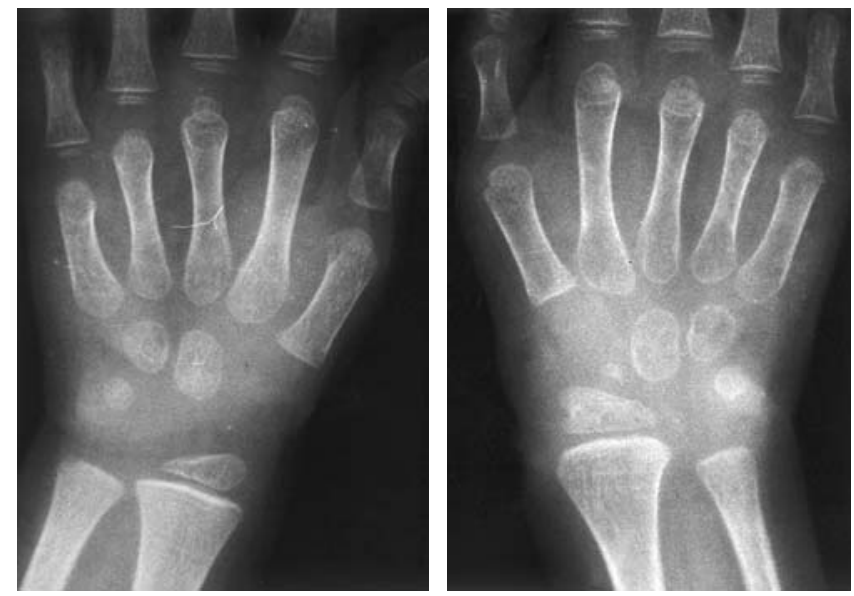

Fig. 4

Anteroposterior radiograph of both wrists in case 3 showing indentation of the radial side of the distal radial epiphysis (the constriction sign) in the right wrist with normal appearances in the opposite left wrist.

for prolonged periods. In children, parents may forget to remove it.

All the cases occurred in an age group where growth is rapid. The red and yellow thread may lie hidden in the wrist crease, especially in infants and children with chubby hands. Due to the rapid growth and increase in the circumference of wrist, the thread cuts through the soft tissue around the wrist. This gradual penetration is surprisingly painless and without neurovascular symptoms. As the skin epithelises over the thread, it can become completely invisible. $^{2}$ This process of slow tissue penetration and rapid healing allows the thread to penetrate tendons and come to lie deep to them as was observed in case 3 (Fig. 5). In long-standing cases the thread may reach the bone producing a 'constriction sign' on plain radiographs.

The clues to this type of embedded foreign body are a thin linear scar, usually encircling the wrist, a non-healing discharging sinus or sinuses and, in long-standing cases, the radiological 'constriction sign'. If the scar lies in one of the wrist creases it may be difficult to detect unless careful attention is paid. The unsuspecting clinician may make a diagnosis of a tuberculous lesion, which is

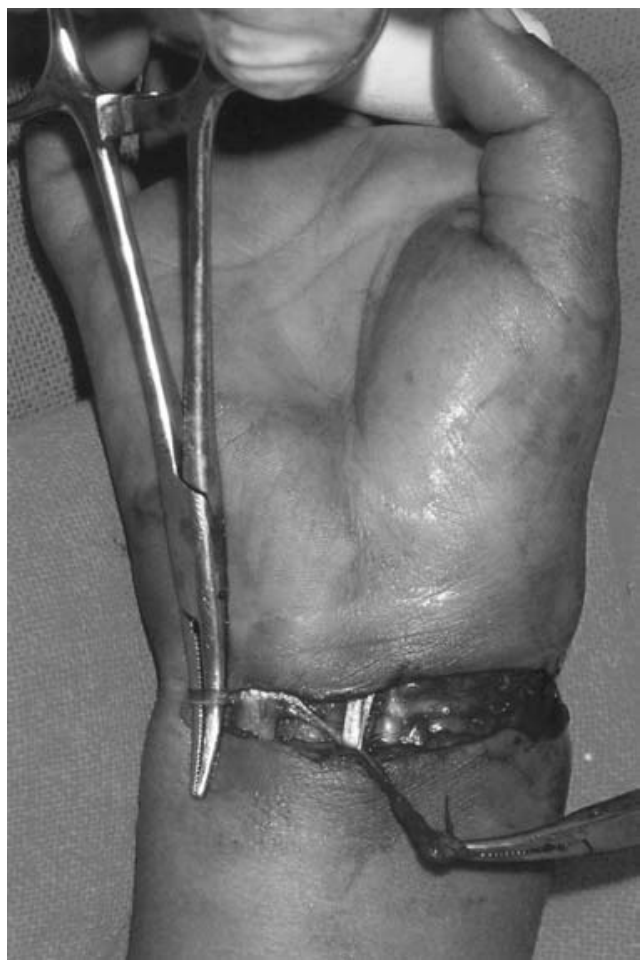

Fig. 5

Intra-operative findings in case 3 showing the thread lying deep to the palmaris longus tendon and partially buried in the flexor carpi ulnaris tendon.

common in India where the disease is endemic. A high index of suspicion, therefore, is necessary to make a correct diagnosis of the Dhaga syndrome.

No benefits in any form have been received or will be received from a commercial party related directly or indirectly to the subject of this article.

\section{References}

1. Tuli SM. General principles of osteoarticular tuberculosis. Clin Orthop 2002;398: $11-9$.

2. Kumar $\mathbf{P}$, Kumar B, Singh $\mathbf{K}$. A constriction ring of the thigh secondary to a rubber band. Plast Reconstr Surg 1995;95:209-10. 